\title{
Heavy Flavor and Quarkonium Production at RHIC and the LHC
}

\section{R. Vogt $\mathbf{t}^{*+}$}

Physics Divsion, Lawrence Livermore National Laboratory, Livermore, CA 94551

Physics Department, University of California at Davis, Davis, CA 95616

E-mail: vogtephysics.ucdavis.edu

\section{R.E. Nelson}

Physics Divsion, Lawrence Livermore National Laboratory, Livermore, CA 94551

Physics Department, University of California at Davis, Davis, CA 95616

\section{A.D. Frawley}

Physics Department, Florida State University, Tallahassee, FL 32306, USA

\begin{abstract}
We assess the theoretical uncertainties on the inclusive charm and $J / \psi$ production cross sections. We use our new results to provide improved baseline comparison calculations at RHIC and the LHC. We discuss cold matter effects on $J / \psi$ production at leading relative to next-to-leading order in the CEM and as a function of rapidity, transverse momentum and centrality. We place these results in the context of the latest RHIC and $\mathrm{LHC}$ results in $p p, p A$ and $A A$ collisions.
\end{abstract}

Xth Quark Confinement and the Hadron Spectrum,

October 8-12, 2012

TUM Campus Garching, Munich, Germany

\footnotetext{
* Speaker.

†The work of R.V. and R.E.N. was performed under the auspices of the U.S. Department of Energy by Lawrence Livermore National Laboratory under Contract DE-AC52-07NA27344 and the JET Collaboration.

${ }^{\ddagger}$ The work of A.D.F. was supported in part by the National Science Foundation Grant NSF PHY-0555660.
} 


\section{Introduction}

Heavy flavor and quarkonium production are complementary processes since the quarkonium cross section is some fraction of the total heavy flavor cross section. At the most basic level, the calculation of the quarkonium production cross section in the color evaporation model [1] is based on parameters set from evaluation of the open heavy flavor cross section. It was shown some time ago that the energy dependence of the open charm and charmonium production cross section was essentially the same, both for photoproduction and hadroproduction [2]. Thus a good understanding of the energy dependence of the charm cross section is important to fix the energy dependence of the $J / \psi$ cross section.

Heavy flavor production is unique in that the total heavy flavor production cross section is analytically calculable to leading order at the partonic level. In addition the finite heavy flavor mass provides a scale to control the transverse momentum distributions down to $p_{T} \rightarrow 0$.

In the absence of any nuclear effects, the heavy flavor cross sections in $p A$ and $A A$ collisions would simply scale with the number of binary collisions. Thus departures from binary scaling for heavy flavor production provide information about nuclear effects. These can be divided into two categories: effects due to embedding the colliding partons in a nucleus (cold matter effects) and effects due to the large energy density in the final state in $A A$ collisions.

The study of dense matter effects requires a detailed understanding of cold matter effects so that they can be unfolded from the dense matter effects. For example, the nuclear suppression factor, $R_{A B}\left(p_{T}, y, b\right)=\left(d \sigma_{A B} / d p_{T} d y\right) /\left(T_{A B}(b) d \sigma_{p p} / d p_{T} d y\right)$, where $A B$ represents $p / \mathrm{d}+A$ or two colliding nuclei and $T_{A B}(b)$ is the nuclear overlap function at impact parameter $b$, depends on both the cold matter effects in $d \sigma_{A B} / d p_{T} d y$ as well as the baseline cross section in $p p$ collisions. If the $p p$ cross section is unmeasured or suffers from poor statistical accuracy, the interpretation of the $A A$ results may be difficult [3].

Previous estimates of the total charm cross section have large uncertainties due to the choice of charm quark mass and the rather wide range of factorization and renormalization scales chosen to delineate the uncertainties $[4,5]$. In this proceeding, we discuss a fit to the total charm cross section data [7] and compare to some relevant heavy flavor data. We then use the fit parameters and their associated uncertainties to determine the uncertainties on the $J / \psi$ cross section. We also show some of our results on cold matter effects on $J / \psi$ production.

\section{Open Heavy Flavor Production}

The total charm cross section calculated in perturbative QCD suffers from large uncertainties due to the choice of quark mass, factorization scale and renormalization scale [5]. Typical lower limits of the factorization and renormalization scales are half the chosen charm quark mass [4]. In this case, the factorization scale is below the minimum scale of the parton densities. In addition, for renormalization scales below $1 \mathrm{GeV}$, the strong coupling constant $\alpha_{s}$ becomes large and the perturbative expansion is unlikely to converge. Thus we seek a set of physically defensible mass and scale parameters that reduce the cross section uncertainty. Because the $J / \psi$ cross sections are calculated with the same set of mass and scale parameters as open charm production in the Color 
Evaporation Model [6], we also place limits on the $J / \psi$ cross section calculated in the CEM for the first time. See Ref. [7] for full details.

The charm quark mass we employ in our calculations is the Particle Data Group (PDG) value based on lattice determinations of the charm quark mass in the $\overline{\mathrm{MS}}$ scheme at $\mu=m: m(m)=$ $1.27 \pm 0.09 \mathrm{GeV}$ [8]. We fit the factorization and renormalization scale parameters to a subset of the fixed target total charm production data with $250 \leq E_{\text {beam }} \leq 920 \mathrm{GeV}$. The data were evaluated and adjusted to the values we employ in our fits using the most up-to-date branching ratios for the measured decay channels in Ref. [9]. We also include data from both PHENIX [10] and STAR $[11,12]$ at $\sqrt{s_{N N}}=200 \mathrm{GeV}$. We neglect unknown next-order uncertainties which could be large for charm where the mass is relatively small and $\mathscr{O}\left(\alpha_{s}^{4}\right)$ corrections could be significant.

The best fit yields the parameter values $m_{c}=1.27 \mathrm{GeV}, \mu_{F} / m=2.1_{-0.85}^{+2.55}$ and $\mu_{R} / m=1.6_{-0.12}^{+0.11}$. We show the $\chi^{2} /$ dof fit contours on the left-hand side of Fig. 1 for $\Delta \chi^{2} /$ dof $=0.3,1$ and 2.3. The one standard deviation uncertainty in the fitted value of $\mu_{F} / m\left(\mu_{R} / m\right)$ was taken as the maximum extent of the $\Delta \chi^{2} /$ dof $=1$ contour along the $\mu_{F} / m\left(\mu_{R} / m\right)$ axis. The one standard deviation uncertainty in the total cross section is the range of cross sections resulting from all combinations of $\mu_{F} / m$ and $\mu_{R} / m$ contained within the $\Delta \chi^{2} /$ dof $=2.3$ contour. The $\Delta \chi^{2} /$ dof $=0.3$ contour is to guide the eye. Note the narrow range in $\mu_{R} / m$ relative to the much broader $\mu_{F} / m$ range. The uncertainty on $\mu_{F} / m$ is larger and very asymmetric. There is a greater uncertainty on the upper limit than the lower limit because there is a much greater change in $x g\left(x, \mu_{F}^{2}\right)$ at lower factorization scales than when $\mu_{F} \gg \mu_{0}$, the minimum scale of the parton densities.
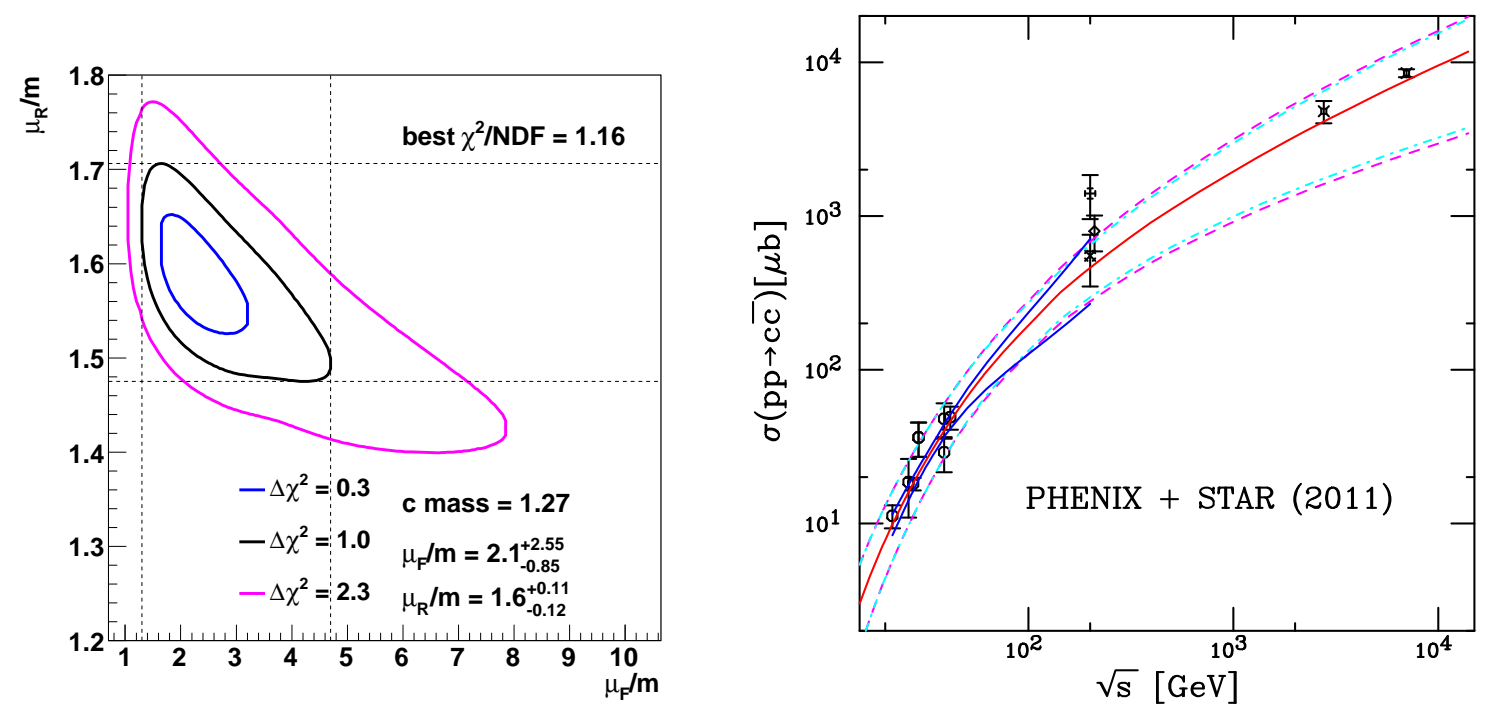

Figure 1: (Left) The $\chi^{2} /$ dof contours for a fit to the fixed target data as well as the PHENIX and STAR cross sections at $\sqrt{s}=200 \mathrm{GeV}$. The best fit values are given for the furthest extent of the $\Delta \chi^{2}=1$ contours. (Right) The energy dependence of the charm total cross section compared to data. The central value of the fit is given by the solid red curve while the dashed magenta curves and dot-dashed cyan curves show the extent of the uncertainty bands, corresponding to the furthest extent of the $\Delta \chi^{2}=1$ contours, see text for details. The solid blue curves in the range $19.4 \leq \sqrt{s} \leq 200 \mathrm{GeV}$ represent the uncertainty obtained from the extent of the $\Delta \chi^{2}=2.3$ contour. 

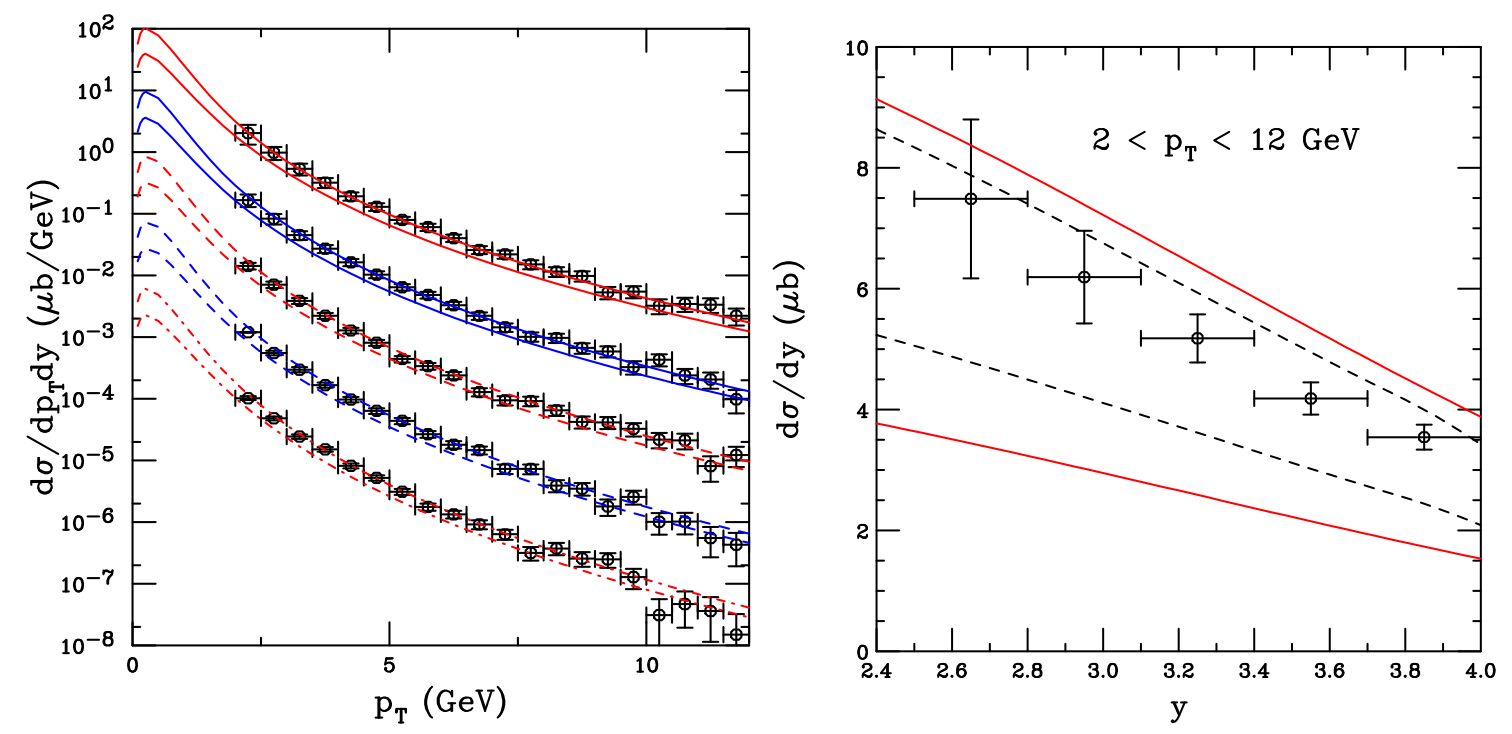

Figure 2: Our calculations are compared with the ALICE inclusive single muon data from heavy flavor decays [13] at the LHC for $p p$ collisions at $\sqrt{s}=7 \mathrm{TeV}$. (Left) The contributions to the $p_{T}$ distributions divided into rapidity bins, from top to bottom: $2.5<y<2.8$ (solid red); $2.8<y<3.1$ (solid blue); $3.1<$ $y<3.4$ (dashed red); $3.4<y<3.7$ (dashed blue); and $3.7<y<4$ (dot-dashed red). The top curves are shown at their calculated value, the others are scaled down by successive factors of 10 to separate them. (Right) The sum of contributions to the rapidity distribution are compared with the FONLL parameter set for charm centered at $m=1.5 \mathrm{GeV}$ [4] (solid red) and our results with $m=1.27 \mathrm{GeV}$ (dashed black).

The right panel of Fig. 1 shows the energy dependence of the total charm cross section for the fits, with the corresponding uncertainty based on results using the one standard deviation uncertainties on the quark mass and scale parameters. If the central, upper and lower limits of $\mu_{R, F} / \mathrm{m}$ are denoted as $C, H$, and $L$ respectively, then the seven sets corresponding to the envelope of the scale uncertainty are $\left\{\left(\mu_{F} / m, \mu_{R} / m\right)\right\}=\{(C, C),(H, H),(L, L),(C, L),(L, C),(C, H),(H, C)\}$. The upper and lower limits on the PDG value of the charm quark mass are 1.36 and $1.18 \mathrm{GeV}$. The uncertainty band can be obtained for the best fit sets by adding the uncertainties from the mass and scale variations in quadrature. The envelope containing the resulting curves,

$$
\begin{aligned}
& \sigma_{\max }=\sigma_{\mathrm{cent}}+\sqrt{\left(\sigma_{\mu, \max }-\sigma_{\mathrm{cent}}\right)^{2}+\left(\sigma_{m, \max }-\sigma_{\mathrm{cent}}\right)^{2}}, \\
& \sigma_{\min }=\sigma_{\mathrm{cent}}-\sqrt{\left(\sigma_{\mu, \min }-\sigma_{\mathrm{cent}}\right)^{2}+\left(\sigma_{m, \text { min }}-\sigma_{\mathrm{cent}}\right)^{2}},
\end{aligned}
$$

defines the uncertainty. The maximum and minimum scale-dependent cross sections, $\sigma_{\mu \text {,max }}$ and $\sigma_{\mu, \min }$, are the largest and smallest cross sections based on the combination of $\mu_{F} / m$ and $\mu_{R} / m$ values given above.

The uncertainty bands are shown for two cases: the region delineated above, similar to Ref. [4], and including the most extreme cases $\left(\mu_{F} / m, \mu_{R} / m\right)=(H, L)$ and $(L, H)$. The difference between the outer magenta curves, which include these extremes, and the cyan curves, which do not, is very small. Therefore, it is reasonable to neglect the extremes. We also show the result for a one standard deviation uncertainty in the total cross section obtained from the $\Delta \chi^{2}=2.3$ contour in 
the blue lines. We have also added the 2.76 and $7 \mathrm{TeV}$ total cross sections obtained by the ALICE collaboration in $p p$ collisions [14], not included in our fits. The calculations are in rather good agreement with the data.

We use the FONLL approach [4] to calculate the heavy flavor semileptonic decay kinematic distributions to compare to single lepton spectra which include $B$ decays as well as $D$ decays. The $B \rightarrow \mu$ and $B \rightarrow D \rightarrow \mu$ bands are calculated with the same mass and scale parameters as in Ref. [4]. The $D \rightarrow \mu$ band is calculated for our best fit parameter set. Figure 2 compares our calculations with the ALICE single muon data in the forward rapidity region, $2.5<y<4$ [13]. The data are given for $2<p_{T}<12 \mathrm{GeV}$ and separated into five rapidity bins, each 0.3 units wide, as shown in the left panel of Fig. 2. The calculations agree well with the measurements over the entire $p_{T}$ range. On the right-hand side of Fig. 2 we present our results in the dashed curves as a function of rapidity, integrated over the same $p_{T}$ range as the data, $2 \leq p_{T} \leq 12 \mathrm{GeV}$. We also show the rapidity distribution obtained using the FONLL charm parameter set with a central charm quark mass of $m=1.5 \mathrm{GeV}$ in red. The $p_{T}$-integrated ALICE data agree well with both calculations. The results with the fitted charm parameter set narrow the uncertainty band without sacrificing consistency with the measured data.

While the agreement between the lepton measurements at RHIC and the LHC and our calculations is encouraging, as noted here and in Ref. [4], there is significant admixture of semileptonic charm and bottom decays, particularly at lepton $p_{T}>4 \mathrm{GeV}$. A better test of our results would be a comparison to open charm hadron data. Thus, in Fig. 3, we show the $D^{0}$ distributions in the ALICE [15] and the LHCb [16] acceptances at midrapidity and forward rapidity respectively as well as the rapidity distribution.
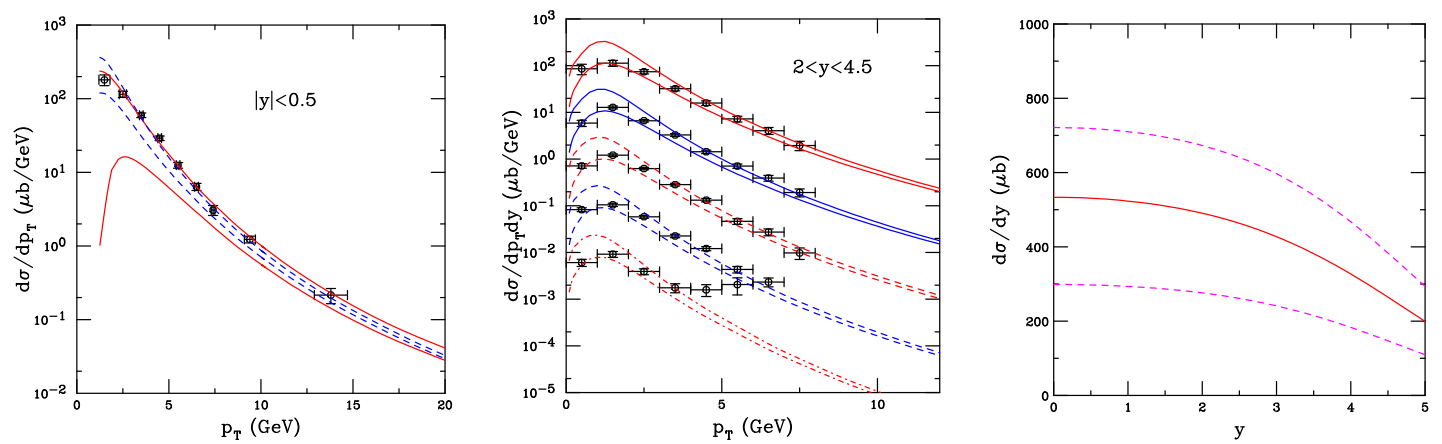

Figure 3: Our calculations are compared with the reconstructed $D^{0}$ meson data at midrapidity [15] (left) and forward rapidity [16] (center) at $\sqrt{s}=7 \mathrm{TeV}$ and as a function of rapidity, integrated over $p_{T}$ (right). At midrapidity the red curves show the uncertainty band based on a charm mass of $1.5 \mathrm{GeV}$ while the results with the present fits are shown in blue. The forward rapidity calculations are shown in the rapidity intervals: $2<y<2.5$ (solid red); $2.5<y<3$ (solid blue); $3<y<3.5$ (dashed red); $3.5<y<4$ (dashed blue); and $4<y<4.5$ (dot-dashed red). The curves are calculated with the charm fit parameters. The sets of results are separated by a factor of 10 between rapidity intervals to facilitate comparison. The lowest rapidity interval, $2<y<2.5$, is not scaled.

The left-hand side of Fig. 3 compares the FONLL calculations with the parameter set based on a charm mass of $1.5 \mathrm{GeV}$ and $0.5 \leq \mu_{F} / m, \mu_{R} / m<1$ (in red) with the fitted parameters based 
on $m=1.27 \mathrm{GeV}$ (in blue) to the ALICE $D^{0}$ meson data at midrapidity. The upper and lower limits of both bands are shown. While the ALICE data are in agreement with the upper limits of both calculations, the large $D$ meson uncertainty is reduced at low $p_{T}$ with the fitted parameter set. The center of Fig. 3 shows the upper and lower limits of the FONLL calculation based on $m=1.27 \mathrm{GeV}$ in the five rapidity intervals of $\Delta y=0.5$ in the range $2<y<4.5$ covered by the LHCb detector. Here also the agreement with the data is very good.

\section{Quarkonium Production}

We now turn to a treatment of quarkonium production within this same framework. In the $\mathrm{CEM}$, the quarkonium production cross section is some fraction, $F_{C}$, of all $Q \bar{Q}$ pairs below the $H \bar{H}$ threshold where $H$ is the lowest mass heavy-flavor hadron. We fit $F_{C}$ to the forward (integrated over $\left.x_{F}>0\right) J / \psi$ cross section data on only $p, \mathrm{Be}, \mathrm{Li}, \mathrm{C}$, and $\mathrm{Si}$ targets. In this way, we avoid uncertainties due to ignoring any cold nuclear matter effects which are on the order of a few percent in light targets. We also restricted ourselves to the forward cross sections only.
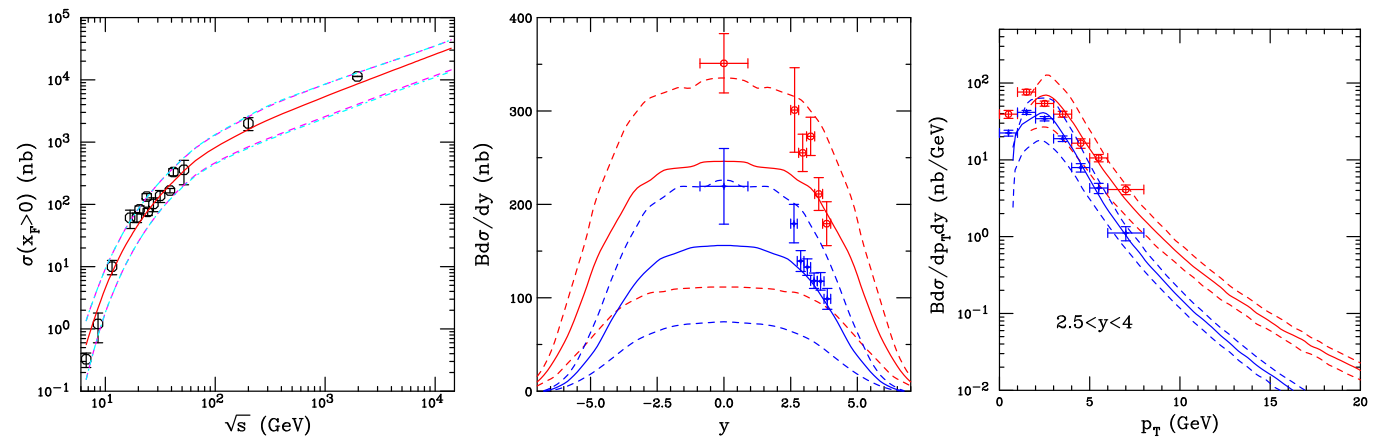

Figure 4: (Left) The uncertainty band on the forward $J / \psi$ cross section calculated based on the $c \bar{c}$ fit. The solid curve is the result based on central value of the open charm fit. The dashed magenta curves and dot-dashed cyan curves show the extent of the corresponding uncertainty bands. (Center) The $J / \psi$ rapidity distributions compared to data from $7 \mathrm{TeV}$ [17] (red points and band) and $2.76 \mathrm{TeV}$ [18] (blue points and band). (Right) The forward $p_{T}$ distributions $(2.5<y<4)$ are also shown. No additional scaling factor has been applied. A $\left\langle k_{T}^{2}\right\rangle$ kick of $1.49 \mathrm{GeV}^{2}(7 \mathrm{TeV})$ and $1.41 \mathrm{GeV}^{2}(2.76 \mathrm{TeV})$ is applied to the $p_{T}$ distributions.

We use the same values of the central charm quark mass and scale parameters as we found for open charm to obtain the $J / \psi$ normalization $F_{C}$ for $\left.\left(m, \mu_{F} / m, \mu_{R} / m\right)=(1.27 \mathrm{GeV}, 2.1,1.6)\right)$. We determine $F_{C}$ only for the above central parameter set and scale the calculations of the open charm cross section with the mass cut by the same value of $F_{C}$ to obtain the extent of the $J / \psi$ uncertainty band. The result is shown on the left-hand side of Fig. 4.

The ALICE 2.76 and $7 \mathrm{TeV}$ inclusive $J / \psi$ rapidity and forward $p_{T}$ distributions $(2.5 \leq y \leq 4)$ are shown in the center and right panels of Fig. 4. The rapidity distribution at $\sqrt{s}=7 \mathrm{TeV}$ is flat over several units of rapidity. The calculated rapidity distribution at $2.76 \mathrm{TeV}$ is not as broad and the agreement with the data is rather good although the midrapidity point remains high relative to the central value of the calculation. The agreement of the calculated $p_{T}$ distributions with the forward rapidity data is quite good with the exception of the lowest $p_{T}$ points where the calculated distributions turn over more quickly than the data. 


\section{Cold Matter Effects}

There are a number of possible cold matter effects on $J / \psi$ production, including modifications of the parton densities in nuclei (shadowing); breakup of the quarkonium state due to inelastic interactions with nucleons (absorption); and energy loss in cold matter. Since the quarkonium absorption cross section decreases with center-of-mass energy, we can expect that shadowing is the most important cold matter effect at midrapidity, see Refs. [19, 20]. Here we show results for the rapidity and $p_{T}$ dependence of shadowing at $\sqrt{s_{N N}}=200 \mathrm{GeV}$ for $\mathrm{d}+\mathrm{Au}$ collisions at RHIC and the rapidity dependence at $\sqrt{s_{N N}}=5 \mathrm{TeV} p+\mathrm{Pb}$ collisions, neglecting absorption. Fig. 5 shows the uncertainty in the shadowing effect due to uncertainties in the EPS09 shadowing parameterization [21] (red) as well as those due to the mass and scale uncertainties obtained in the fit to the total charm cross section (blue) calculated with the EPS09 central set. All the calculations are next-toleading order in the total cross section. The EPS09 band is obtained by calculating the deviations from the central value for the 15 parameter variations on either side of the central set and adding them in quadrature. With the new uncertainties on the charm cross section, the band obtained with the mass and scale variation is narrower than that with the EPS09 variations.
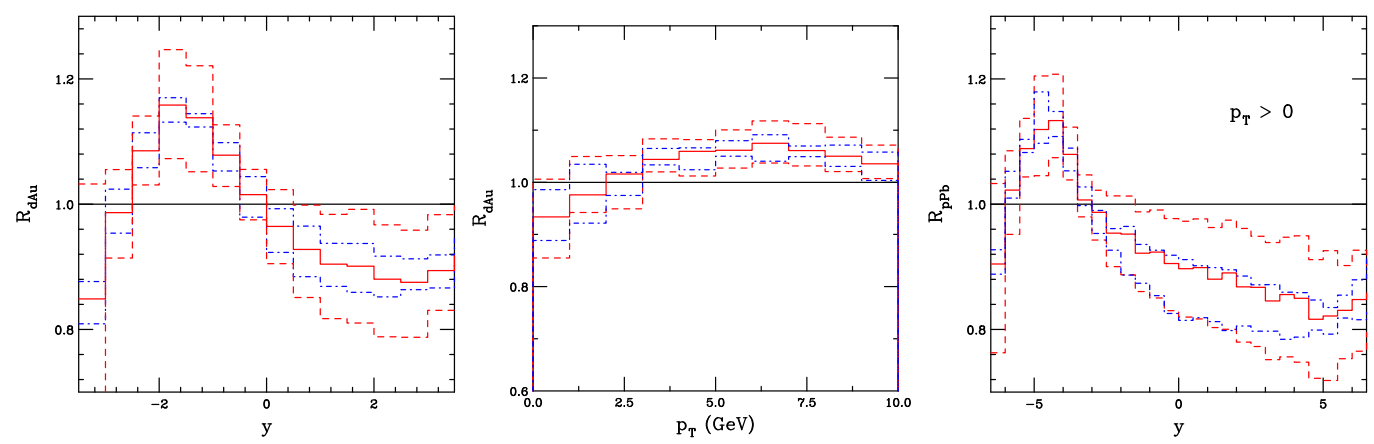

Figure 5: The ratio $R_{\mathrm{dAu}}$ calculated with the central EPS09 set and central mass and scale parameters (red). The variation due to mass and scale choice is given by the blue dot-dashed histograms while the variation in the EPS09 parameterization with the central mass and scale values is shown by the red dashed histograms. The left and center panels give the result as a function of rapidity and $p_{T}$ respectively for $\sqrt{s_{N N}}=200 \mathrm{GeV}$ $\mathrm{d}+\mathrm{Au}$ collisions while the right panel shows the result as a function of rapidity for $\sqrt{s_{N N}}=5 \mathrm{TeV} p+\mathrm{Pb}$ collisions.

\section{Summary}

We have narrowed the uncertainty band on the open heavy flavor cross section and, in so doing, have also provided a realistic uncertainty band on $J / \psi$ production in the Color Evaporation Model. While the fits have been made by comparing the calculated NLO charm production cross section to available data at fixed-target energies and at RHIC, they are in good agreement with the extracted total charm cross sections at the LHC. The same parameter set also provides good agreement with the distributions of single leptons from semileptonic heavy flavor decays at RHIC and the LHC as well as the reconstructed $D$ meson distributions. 
We have used the same fit parameters in the calculation of $J / \psi$ production in the color evaporation model and have thus provided the first uncertainty band on $J / \psi$ production in this approach. The energy dependence of the total $J / \psi$ cross section that results is a good match to the data up to collider energies. The $p_{T}$ distributions are also in good agreement with the data from RHIC and the LHC.

Finally, we have shown uncertainties in shadowing effects on $J / \psi$ production as a function of rapidity and transverse momentum. The uncertainties resulting from the mass and scale parameters are smaller than those due to gluon shadowing.

\section{References}

[1] A. D. Frawley, T. Ullrich and R. Vogt, Phys. Rept. 462 (2008) 125 [arXiv:0806.1013 [nucl-ex]].

[2] J. F. Amundson, O. J. P. Eboli, E. M. Gregores and F. Halzen, Phys. Lett. B 390 (1997) 323 [arXiv:hep-ph/9605295].

[3] T. Dahms (CMS Collaboration), to be published in the proceedings of the $5^{\text {th }}$ Int. Conf. on Hard and Electromagnetic Probes of High-Energy Nuclear Collisions, arXiv:1209.3361 [nucl-ex].

[4] M. Cacciari, P. Nason and R. Vogt, Phys. Rev. Lett. 95, 122001 (2005).

[5] R. Vogt, Eur. Phys. J. ST 155, 213 (2008) [arXiv:0709.2531 [hep-ph]].

[6] R. Gavai, D. Kharzeev, H. Satz, G. A. Schuler, K. Sridhar and R. Vogt, Int. J. Mod. Phys. A 10, 3043 (1995).

[7] R. E. Nelson, R. Vogt and A. D. Frawley, arXiv:1210:xxx [hep-ph].

[8] C. McNeile, C. T. H. Davies, E. Follana, K. Hornbostel and G. P. Lepage, Phys. Rev. D 82, 034512 (2010).

[9] C. Lourenço and H. K. Wöhri, Phys. Rept. 433, 127 (2006) [hep-ph/0609101].

[10] A. Adare et al. [PHENIX Collaboration], Phys. Rev. Lett. 97, 252002 (2006).

[11] Y. Zhang et al. [STAR Collaboration], J. Phys. G 38, 124142 (2011).

[12] L. Adamczyk et al. [STAR Collaboration], arXiv:1204.4244 [nucl-ex] - pub info??.

[13] B. Abelev et al. [ALICE Collaboration], arXiv:1201.3791 [hep-ex].

[14] B. Abelev et al. [ALICE Collaboration], arXiv:1205.4007 [hep-ex].

[15] B. Abelev et al. [ALICE Collaboration], JHEP 1201, 128 (2012) [arXiv:1111.1553 [hep-ex]].

[16] M. Schmelling and P. Spradlin [LHCb Collaboration], LHCb-CONF-2010-013.

[17] K. Aamodt et al. [ALICE Collaboration], Phys. Lett. B 704, 011 (2011).

[18] B. Abelev et al. [ALICE Collaboration], arXiv:1203.3641 [hep-ex].

[19] C. Lourenço, R. Vogt and H. Wöhri, JHEP 0902, 014 (2009) [arXiv:0901.3054 [hep-ph]].

[20] D. C. McGlinchey, A. D. Frawley and R. Vogt, arXiv:12XX.yyyy.

[21] K. J. Eskola, H. Paukkunen and C. A. Salgado, JHEP 0904, 065 (2009) [arXiv:0902.4154 [hep-ph]]. 